\title{
Controllable Fabrication of Ultrathin Carbon Layer-Wrapped Noble Metal Nanoparticles Based on Laser Ablation in Carbon- Contained Solutions
}

\author{
Yebin $\mathrm{Cai}^{1,2}$ and Liaoping $\mathrm{Cai}^{1^{*}}$ \\ ${ }^{1}$ Agricultural Machinery Appraisal Station, Hubei University of Technology, Wuhan, 430068, P.R.China \\ ${ }^{2}$ Instutute of Geodesy, University of Stuttgart, 70174 Stuttgart, Germany \\ Email: liaopingcai19630163.com
}

\begin{abstract}
A simple and flexible method is presented for fabricating the $\mathrm{Au}$ or $\mathrm{Ag}$ nanoparticles (NPs) coated with an ultrathin layer of carbon via laser ablation of metal targets in carbon-contained solutions. After the ablation, $\mathrm{Au}$ and $\mathrm{Ag}$ NPs (about 20nm in mean size) are wrapped with an ultrathin layer of graphite-like carbon. Typically, ablation of $\mathrm{Au}$ or $\mathrm{Ag}$ metal target in toluene contained solution can form carbon shell-wrapped $\mathrm{Au}$ or $\mathrm{Ag}$ NPs with the homogeneous shell's thickness. Further experiments have revealed that the carbon shell's thickness depends mainly on the carbon content in solutions and can be tuned from $2 \mathrm{~nm}$ down to $0.5 \mathrm{~nm}$, while laser ablation power and duration only insignificantly influence the thickness. The formation of the wrapped NPs is well described by the metal NPs' generation and then carbon-deposition during the ablation. This work provides a flexible route for wrapping the metal NPs with the ultrathin shell-layer.
\end{abstract}

Keywords: Ultrathin carbon layer-wrapped metal nanoparticles, one step synthesis route, laser ablation in carbon-contained solutions.

\section{Introduction}

The noble metal nanoparticles (NPs) have been attracting much attention due to their unique properties, and have many applications, in catalysis [1], optical-electronic devices [2], optical sensors [3] and medicine [4] and so on. For some practical applications, surface-coating or wrapping is necessary to isolate the metal NPs from environment. For example, noble metal NPs can be used for the excellent substrates of surface-enhanced Raman scattering (SERS) sensors due to their surface plasmon resonance, which has been extensively reported [5-8]. For some target test molecules, the films $[9,10]$ or arrays [1114] consisting of the noble metal NPs can be used, without any surface coating, as SERS substrates for detecting them. However, for the gas molecules which cannot be adsorbed on the metal NPs, or even if these molecules can be adsorbed on the metal NPs, catalysis or charge transfer between them during laser excitation would occur, it is invalid to directly use the noble metal NPs as SERS substrates. Obviously, if these metal NPs are coated or wrapped with a thin layer of material which can not only isolate the NPs from the test molecules but also adsorb or capture them, the above issue could be solved. Importantly, this wrapping shell should be thin $(\sim 1 \mathrm{~nm}$ or less $)$ and uniform enough in thickness for the SERS-based detection [15]. Otherwise, over-thick shell would induce weak or even no SERS effect because the enhancement effect of local electromagnetic field is mainly confined within the nanoscaled space above the noble metal NPs.16 There have been many methods developed for coating the NPs, such as sol-gel coating [16], solution dipping [17, 18], vapor deposition [19-21], etc. However, using these methods, it is difficult to obtain the ultrathin (or nanoscaled thin) and homogeneous coating layer. The controllable fabrication of the uniform and ultra-thin wrapping layer on the noble metal NPs is still expected and in challenge.

In this study, we present a simple and flexible route for fabricating the ultrathin carbon-wrapped noble metal ( $\mathrm{Au}$ and $\mathrm{Ag}$ ) NPs just via laser ablation of $\mathrm{Au}$ or $\mathrm{Ag}$ targets in the carbon-contained solutions. Carbon is used as wrapping material due to its relatively high chemical and thermal stability [22-24]. It can protect the metal NPs from oxidation and agglomeration, but could adsorb many target molecules and possess good biocompatibility and non-catalysis. The uniform and ultrathin carbon layerwrapped $\mathrm{Au}$ or $\mathrm{Ag}$ NPs have been obtained via laser-ablating metal targets in carbon-contained 
solutions, Typically, for ablation in toluene-contained ethanol solution, the carbon wrapping is controllable in thickness from $2 \mathrm{~nm}$ down to $\sim 0.5 \mathrm{~nm}$, depending on the toluene content in the solution. It has been found that the wrapping-layer' thickness can be tuned mainly by carbon content in solutions. This work presents an effective route to the ultrathin shell-wrapped metal NPs.

\section{Experiments}

A gold (or silver) metal plate or target $(1.5 \mathrm{~cm} \times 1 \mathrm{~cm}$ in size and $99.99 \%$ in purity) was fixed on a bracket immersed in a quartz glass cup with $10 \mathrm{~mL}$ of toluene-contained ethanol solution (the ratio of toluene to ethanol was 1:9 in volume). The cup was put on a table. The solution in the cup was stirred continuously, and the metal plate immersed in the solution was ablated by a Nd:YAG pulse laser (10 ns in pulse width, $1064 \mathrm{~nm}$ in wavelength and $10 \mathrm{~Hz}$ in frequency) with $60 \mathrm{~mJ} /$ pulse in power and $2 \mathrm{~mm}$ in spot size on the metal plate, as schematically in Figure S1. After ablation for 30 min, colloidal solution was obtained in the cup. In addition, the Au or Ag plate in water was also ablated for comparison. The optical absorption spectral measurements were conducted for the ablated solutions on a Cary-5E UVvis-NIR spectrophotometer. The morphology and microstructure for the metal NPs in the ablated solutions were examined on a field emission scanning electron microscope (FESEM, FEI Sirion 200) and transmission electron microscope (TEM, JEM-200CX). The Raman spectra were measured on a Raman spectrometer (Renishaw in Via Reflex) using a laser beam with $1 \mathrm{~mW}$ in power, $532 \mathrm{~nm}$ in wavelength, and $5 \mu \mathrm{m}$ in spot size.

\section{$3 \quad$ Results and Discussion}

After laser-ablating a Au target in water or the toluene-contained ethanol solution, Au colloidal solution was obtained. The optical absorbance spectra are shown in Figure 1. For ablation in water, an optical absorbance peak was observed at $520 \mathrm{~nm}$, which correspond to the surface plasmon resonance (SPR) of $\mathrm{Au}$ NPs [25-28] and indicate formation of Au NPs in water after ablation. In contrary, ablation in the toluene-contained ethanol solution only results in a much weaker but broader SPR peak at about 555 $\mathrm{nm}$, as illustrated in Fig.1A. Also, if using Ag target, the ablation in water induces a strong SPR of Ag NPs at $400 \mathrm{~nm}$, while the only a broad absorption band with much lower intensity at $420 \mathrm{~nm}$ was observed after ablation in the toluene-contained ethanol solution, as shown in Fig.1B. The ablation in the toluene-contained solution results in the significant decrease of SPR in intensity and red-shift in position.
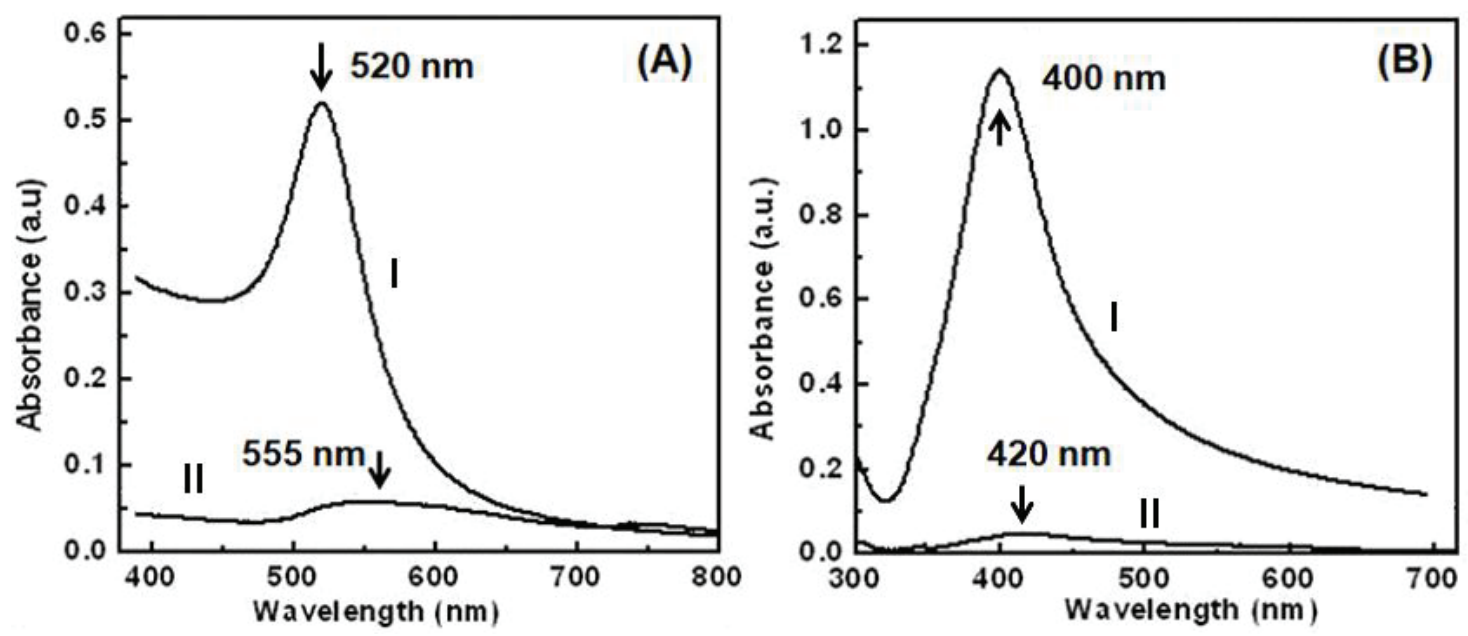

Figure 1. The absorbance spectra of the colloidal solutions obtained after laser ablation of $\mathrm{Au}(\mathrm{A})$ and $\mathrm{Ag}$ (B) targets in (I) water and (II) the toluene-contained ethanol solution. 


\subsection{Morphology and Structure}

Correspondingly, TEM examination has revealed that the Au NPs were formed with size smaller than $20 \mathrm{~nm}$ after ablating Au target in water (see Fig. S2). Contrarily, after ablation in the toluene-contained solution, the ultra-thin coating-wrapped Au NPs with the mean size of $20 \mathrm{~nm}$ were obtained, showing core-shell structure, as illustrated in Fig.2A, B. The high resolution TEM has confirmed that the core parts in the wrapped NPs are Au crystal (not shown here). The coating is about $2 \mathrm{~nm}$ in thickness with layered structure of $0.37 \mathrm{~nm}$ in spacing (Fig.2C). Such spacing is close to that of (001) plane in bulk graphite $(0.34 \mathrm{~nm})$. If ablating $\mathrm{Ag}$ target in the toluene-contained solution, we could correspondingly obtain the wrapped Ag NPs with $15 \mathrm{~nm}$ in mean size and about $2 \mathrm{~nm}$ in shell thickness. The morphology is similar to that shown in Fig.2, as illustrated in Fig.3.
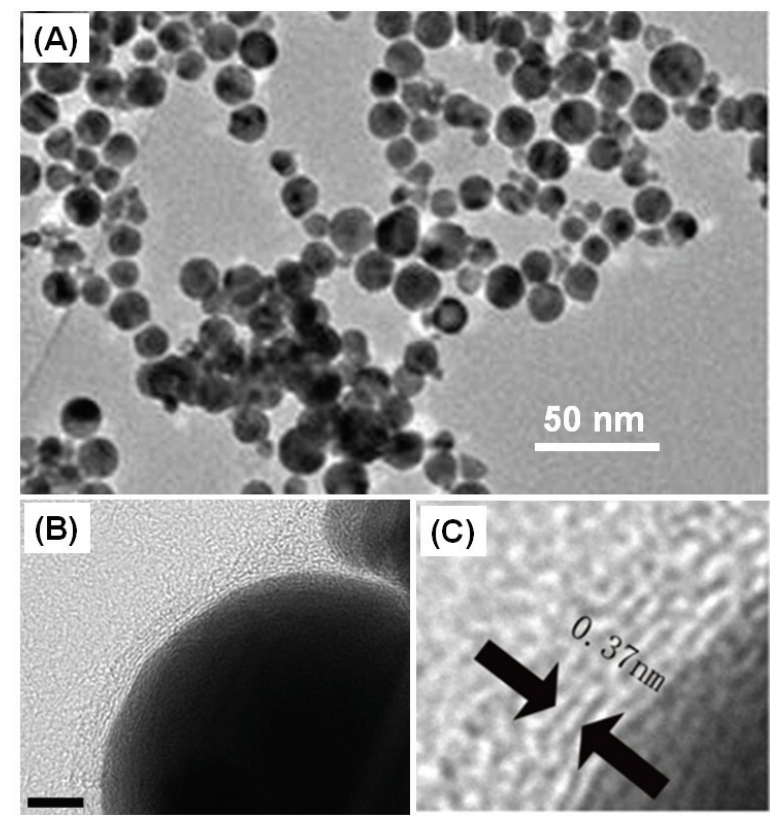

Figure 2. The TEM observations of the particles obtained after laser-ablating Au target in the toluene-contained solution. (A): TEM image with low magnification. (B): The TEM image of a single particle, showing core-shell structure. The scale bar is $5 \mathrm{~nm}$. (C): The high resolution TEM image of the shell layer.
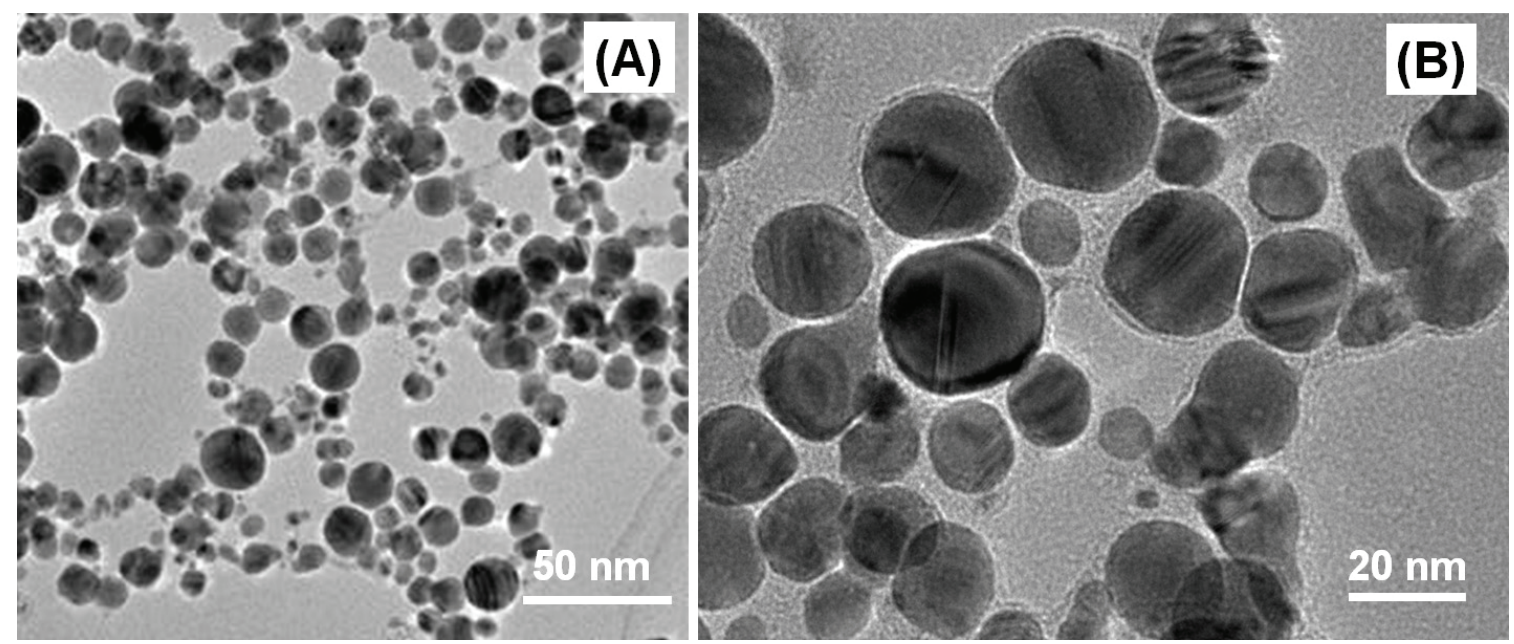

Figure 3. The TEM observations of the particles obtained after laser-ablating Ag target in the toluene-contained solution. (A) and (B): TEM images with low and high magnifications respectively. The shell thickness is about 2 $\mathrm{nm}$. 
Further, Raman spectral measurement was carried out for the structural information of the wrapping layer on the metal NPs. The wrapped Au NPs' film was prepared on an ITO glass via electrophoretic deposition (Fig.S3), as previously reported [29-31], before Raman measurement. Fig.4 shows the corresponding result. The broad peaks around $1570 \mathrm{~cm}^{-1}$ and $1350 \mathrm{~cm}^{-1}$ are assigned to $\mathrm{G}$ and D bands of graphitic carbon, respectively [32-34]. The Raman spectrum is also similar for the wrapped Ag NPs. Obviously, it is such carbon coating that the SPR of the Au or Ag NPs is significantly suppressed.

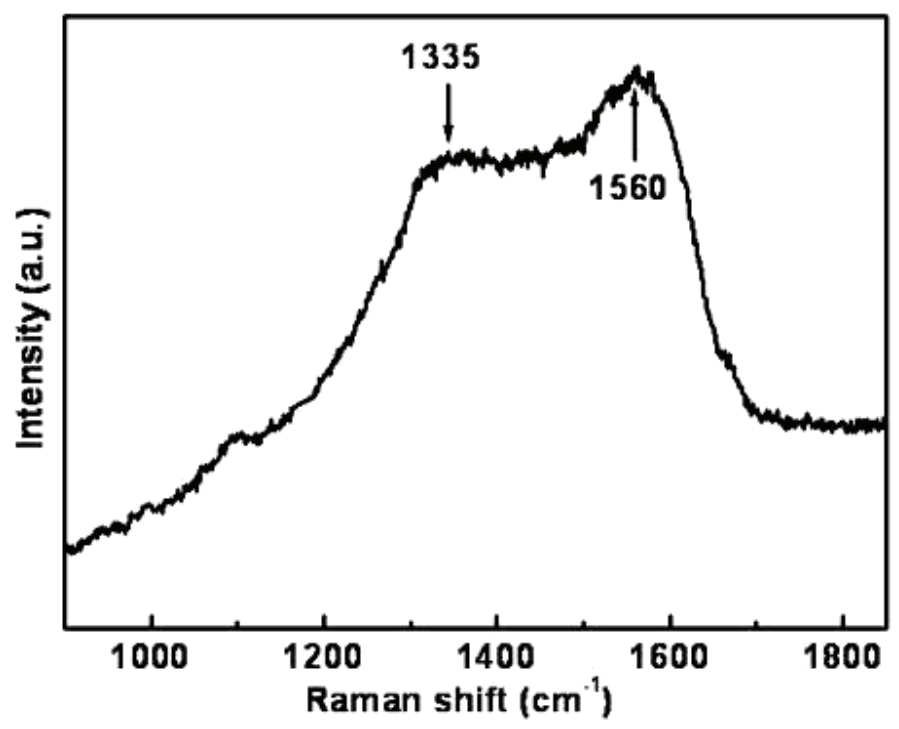

Figure 4. Raman spectrum of the wrapped Au NPs.

Finally, the $\mathrm{Au}$ NPs are controllable in size by the laser ablation power. In our experimental conditions, the mean size of NPs decreases from $>20 \mathrm{~nm}$ to about $10 \mathrm{~nm}$ with increasing laser power from 40 to $100 \mathrm{~mJ} /$ pulse, as illustrated in Fig. S4. But, the wrapping-layer is nearly unchanged in thickness $(\sim 2 \mathrm{~nm})$ with the laser power.

\subsection{Influence of the Solution Composition}

It has been revealed that the NPs' amount in the solution increases with the ablation time, as expected, but the carbon wrapping layer is nearly unchanged in thickness when the ablation was longer than 2 min, and remains to be about $2 \mathrm{~nm}$, as shown in Fig.2. However, the coating's thickness is mainly determined by content of the carbon component in the solution. The high carbon content in solution would induce the thick carbon coating, or vice versa.
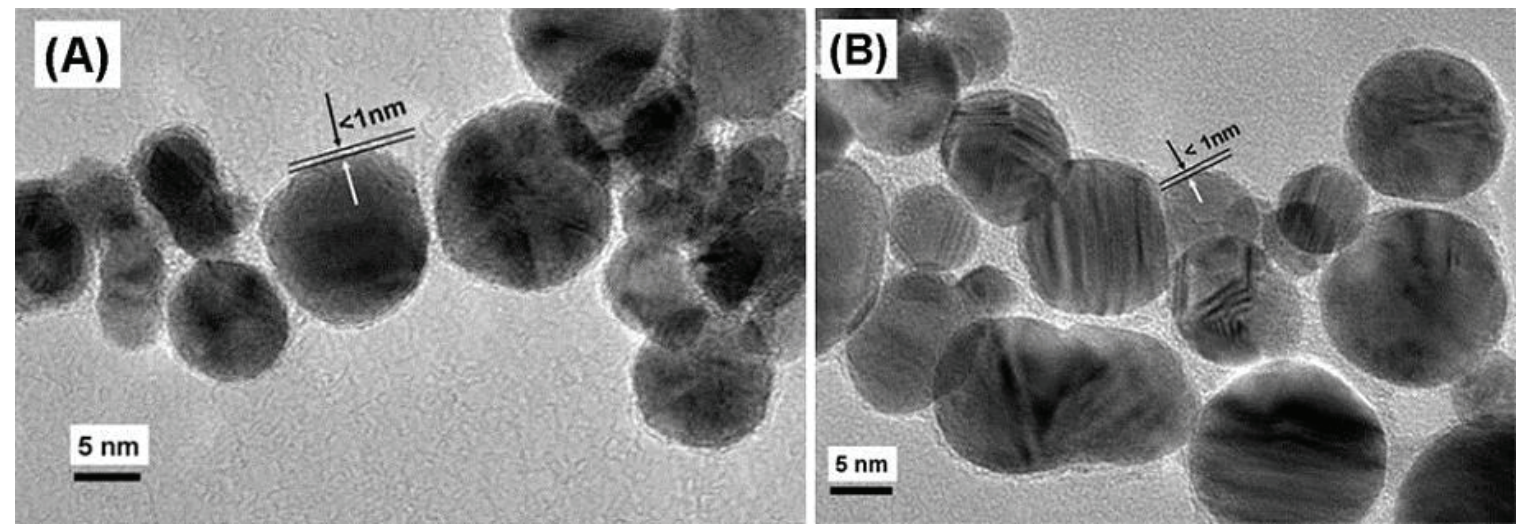

Figure 5. The TEM images of the products obtained after laser-ablating (A) Au and (B) Ag targets in the pure ethanol solution without toluene, showing formation of the ultrathin carbon shells. 
If laser-ablating the $\mathrm{Au}$ or $\mathrm{Ag}$ target in the pure ethanol solution without toluene, we could also obtain the wrapped $\mathrm{Au}$ or $\mathrm{Ag} \mathrm{NPs}$, but the wrapping layer was much thinner and only $\sim 0.5 \mathrm{~nm}$ in thickness, as shown in Fig.5. The Raman spectral measurements have confirmed that the wrapping layer is carbon. The spectrum is similar to that shown in Fig.4. The carbon wrapping layer on Au or Ag NPs increases in thickness with increasing toluene content in the ethanol solution.

In addition to the ethanol or toluene-contained ethanol solution, the other carbon-contained solutions, such as methanol, are also suitable for fabricating the noble metal NPs coated with the ultrathin carbon layer via laser ablation. It means that the thickness could be well controlled by the carbon content in solution.

\subsection{Formation of the Carbon-Wrapped Metal NPs}

The formation of the metal NPs wrapped with carbon shell is easily understood. Briefly, when a pulse laser beam was irradiated on a metal ( $\mathrm{Au}$ or $\mathrm{Ag}$ ) target in carbon-contained solution, the metal plasma plume with high temperature and high-pressure would be generated instantly on the interface between the metal and solution [35-37]. Such metal plasma plume would adiabatically expand ultrasonically, resulting in cooling of the metal plasma and formation of metal NPs. Meanwhile, C-C and C-O bonds in toluene and ethanol molecules, on the interface between the carbon-contained solution and the metal plasma plume, would be broken to produce carbon atoms due to the laser-induced extreme local environment. The produced carbon atoms could be deposited on the pre-formed Ag or Au NPs to generate graphite-like wrapping shell.

On this basis, the thickness of the wrapping layer depends on the capability of the solution to produce carbon atoms. So, the carbon-abundant solutions, such as toluene-contained ethanol solutions can induce the thicker carbon wrapping layer than the pure ethanol solution. Only the laser ablation in the solutions with low carbon content can produce the ultrathin carbon layer-coated NPs.

As mentioned above, the wrapping shell's thickness is nearly independent of the laser ablation power and duration. This originates from the balance between the laser ablation-induced $\mathrm{C}$ atoms and metal NPs in number. It means that the higher laser power or the longer ablation would not only generate the more carbon atoms in the solutions but also produce the more Ag or $\mathrm{Au} \mathrm{NPs.} \mathrm{In} \mathrm{our} \mathrm{experimental}$ conditions, both are nearly balanced, leading to the ablation parameter-independent thickness of the carbon shell.

\section{Conclusion}

In summary, an effective route has been used for fabricating the ultrathin carbon layer-wrapped Au or Ag NPs via pulse laser ablation in carbon-contained solutions. The pulse laser irradiation on a metal target in the solution would not only instantly form the metal plasma, which induces formation of metal NPs, but also generate carbon atoms by breaking $\mathrm{C}-\mathrm{C}$ and $\mathrm{C}-\mathrm{O}$ bonds in solution, that results in carbon deposition and hence graphite-like carbon layer wrapping on the metal NPs. The wrapping layer could be controlled and tuned in the thickness just by carbon content in solutions. Typically, for ablation in toluene-contained ethanol solution, we can obtain the carbon-wrapped Ag or Au NPs with the thickness from about $2 \mathrm{~nm}$ down to $\sim 0.5 \mathrm{~nm}$ depending on the toluene content in the solution. This study could be also suitable for the ultrathin shell layer-wrapped metal NPs of the other materials.

\section{References}

1. J. M. Campelo, D. Luna, R. Luque, J. M. Marinas and A. A. Romero, "Sustainable preparation of supported metal nanoparticles and their applications in catalysis," ChemSusChem, vol. 2, pp. 18- 45, 2009.

2. G. V. Hartland, "Optical Studies of Dynamics in Noble Metal Nanostructures," Chem. Rev., vol. 111, pp. 3858$3887,2011$.

3. I. H. El-Sayed, X. Huang and M. A. El-Sayed, "Surface plasmon resonance scattering and absorption of antiEGFR antibody conjugated gold nanoparticles in cancer diagnostics: Applications in oral cancer," Nano Lett, vol. 5, pp. 829-834, 2005. 
4. H. Park, H. Tsutsumi and H. Mihara, "Cell penetration and cell-selective drug delivery using a -helix peptides conjugated with gold nanoparticles," Biomaterials., vol.34, pp. 4872- 4879, 2013.

5. S. Nie and S. R. Emory, "Probing single molecules and single nanoparticles by surface-enhanced Raman scattering," Science, vol. 275, pp. 1102-1106, 1997.

6. J. Kneipp, H. Kneipp, B. Wittig and K. Kneipp, "Novel optical nanosensors for probing and imaging live cells" Nanomed.-Nanotechn. Biology and Med., vol. 6, pp.214-226 , 2010.

7. S. J. Lee, A. R. Morrill and M. Moskovits, "Hot spots in silver nanowire bundles for surface-enhanced Raman spectroscopy," J. Am. Chem. Soc., vol.128, pp. 2200-2201, 2006.

8. Y. J. Xiong, J. M. McLellan, J. Y. Chen, Y. D. Yin, Z. Y. Li and Y. N. Xia, "Kinetically controlled synthesis of triangular and hexagonal nanoplates of palladium and their SPR/SERS properties," J. Am. Chem. Soc., vol. 127, pp. 17118 - 17127, 2005.

9. L. A. Dick, A. D. McFarland, C. L. Haynes and R. P. Van Duyne, "Metal film over nanosphere (MFON) electrodes for surface-enhanced Raman spectroscopy (SERS): Improvements in surface nanostructure stability and suppression of irreversible loss," J. Phys. Chem. B, vol. 106, pp. 853- 860, 2001

10. G. Upender, R. Satyavathi, B. Raju, K. Shadak Alee, D. Narayana Rao and C. Bansal, "Silver nanocluster films as novel SERS substrates for ultrasensitive detection of molecules," Chem. Phys. Lett., vol. 511, pp. 309-314, 2011.

11. J. Wang, G. Duan, Y. Li, G. Liu, Z. Dai, H. Zhang and W. Cai, "An invisible template method toward gold regular arrays of nanoflowers by electrodeposition," Langmuir, vol. 29, pp. 3512- 3517, 2013.

12. Y. Li, W. Cai and G. Duan, "Ordered micro/nanostructured arrays based on the monolayer colloidal crystals," Chem. Mater., vol. 20, pp. 615-624, 2007.

13. G. T. Duan, W. P. Cai, Y. Y. Luo, Y. Li and Y. Lei, "Hierarchical surface rough ordered Au particle arrays and their surface enhanced Raman scattering," Appl. Phys. Lett., vol. 89, pp. 181918, 2006.

14. G. Liu, W. Cai, L. Kong, G. Duan, Y. Li, J. Wang, G. Zuo and Z. Cheng, "Standing Ag nanoplate-built hollow microsphere arrays: Controllable structural parameters and strong SERS performances," J. Mater. Chem., vol. 22, pp. 3177- 3184, 2012.

15. F. W. King, R. P. Vanduyne and G. C. Schatz, "Theory of Raman-scattering by molecules adsorbed on electrode surfaces," J. Chem. Phys., vol. 69, pp. 4472-4481, 1978.

16. J. F. Li, Y. F. Huang, Y. Ding, Z. L. Yang, S. B. Li, X. S. Zhou, F. R. Fan, W. Zhang, Z.Y. Zhou, D. Y. Wu, B. Ren, Z. L. Wang, Z. Q. Tian, "Shell-isolated nanoparticle-enhanced Raman spectroscopy," Nature, vol. 464, pp. 392- 395, 2010.

17. S. Malynych, I. Luzinov and G. Chumanov, "Poly(vinyl pyridine) as a universal surface modifier for iImmobilization of nanoparticles," J. Phys. Chem. B, vol. 106, pp. 1280-1285, 2002.

18. L. Gao and J. He, "A facile dip-coating approach based on three silica sols to fabrication of broadband antireflective superhydrophobic coatings," J. Colloid Interf. Sci, vol. 400, pp. 24- 30, 2013.

19. A. M. Boies, J. T. Roberts, S. L. Girshick, B. Zhang, T. Nakamura and A. Mochizuki, "SiO2 coating of silver nanoparticles by photoinduced chemical vapor deposition, " Nanotechnology, vol. 20, pp. 295604, 2009.

20. A. M. Boies, P. Lei, S. Calder and S. L. Girshick, "Gas-phase production of gold-decorated silica nanoparticles, " Nanotechnology, vol. 22, pp. 315603, 2011.

21. M. Seipenbusch and A. Binder, "Structural stabilization of metal nanoparticles by chemical vapor depositionapplied silica coatings," J. Phys. Chem. C, vol. 113, pp. 20606-20610, 2009.

22. D. S. Su, S. Perathoner and G. Centi, "Nanocarbons for the development of advanced catalysts," Chem. Rev., vol. 113, pp. 5782-5816, 2013.

23. S. Kundu, Y. Wang, W. Xia and M. Muhler, "Thermal stability and reducibility of oxygen-containing functional groups on multiwalled carbon nanotube surfaces: A quantitative high resolution XPS and TPD/TPR study," $J$. Phys. Chem. C, vol. 112, pp. 16869- 16878, 2008.

24. J. J. Delgado, R. Vieira, G. Rebmann, D. S. Su, N. Keller, M. J. Ledoux and R. Schlögl, "Supported carbon nanofibers for the fixed-bed synthesis of styrene," Carbon, vol. 44, pp. 809- 812, 2006.

25. K.-S. Lee and M. A. El-Sayed, "Gold and silver nanoparticles in sensing and imaging: sensitivity of plasmon response to size, shape, and metal composition," J. Phys. Chem. B, vol. 110, pp. 19220-19225, 2006.

26. G. V. Bianco, M. M. Giangregorio, M. Losurdo, P. Capezzuto and G. Bruno, "Supported faceted gold nanoparticles with tunable surface plasmon resonance for NIR-SERS," Adv. Funct. Mater., vol. 22, pp. 50815088, 2012. 
27. J. Y. Chen and Y. C. Chen, "A label-free sensing method for phosphopeptides using two-layer gold nanoparticlebased localized surface plasma resonance spectroscopy," Analyt. and Bioanalyt.l Chem., vol. 399, pp. 1173- 1180, 2011.

28. T. J. Norman, C. D. Grant, D. Magana, J. Z. Zhang, J. Liu, D. L. Cao, F. Bridges and A. Van Buuren, "Near infrared optical absorption of gold nanoparticle aggregates," J. Phys. Chem. B, vol. 106, pp.7005- 7012, 2002.

29. S. Yang, W. Cai, G. Liu and H. Zeng, "From nanoparticles to nanoplates: preferential oriented connection of Ag colloids during electrophoretic deposition," J. Phys. Chem. C, vol. 113, pp. 7692- 7696, 2009.

30. S. K. Yang, W. P. Cai, J. L. Yang and H. B. Zeng, "General and simple route to micro/nanostructured hollowsphere arrays based on electrophoresis of colloids induced by laser ablation in liquid," Langmuir, vol. 25, pp. 8287-8291, 2009.

31. H. He, W. P. Cai, Y. X. Lin and B. S. Chen, "Au nanochain-built 3D netlike porous films based on laser ablation in water and electrophoretic deposition," Chem. Commun., vol. 46, pp. 7223- 7225, 2010.

32. R. J. Nemanich and S. A. Solin, "First- and second-order Raman scattering from finite-size crystals of graphite," Phys. Rev. B, vol. 20, pp. 392- 401,1979.

33. F. Tuinstra and J. L. Koenig, "Raman spectrum of graphite," J. Chem. Phys., vol. 53, pp.1126- 1126, 1970.

34. B. O. Boskovic, V. Stolojan, R. U. A. Khan, S. Haq and S. R. P. Silva, "Large-area synthesis of carbon nanofibres at room temperature," Nature Materials, vol. 1, pp. 165- 168, 2002.

35. P. P. Patil, D. M. Phase, S. A. Kulkarni, S. V. Ghaisas, S. K. Kulkarni, S. M. Kanetkar, S. B. Ogale and V. G. Bhide, "Pulsed-laser-induced reactive quenching at liquid-solid interface: Aqueous oxidation of iron," Phys. Rev. Lett., vol. 58, pp. 238- 241, 1987.

36. F. Mafune, J. Kohno, Y. Takeda, T. Kondow and H. Sawabe, "Formation of gold nanoparticles by laser ablation in aqueous solution of surfactant," J. Phys. Chem. B, vol. 105, pp. 5114- 5120, 2001.

37. C.-C. Huang, C.-S. Yeh and C.-J. Ho, "Laser ablation synthesis of spindle-like gallium oxide hydroxide nanoparticles with the presence of cationic cetyltrimethylammonium bromide," J. Phys. Chem. B, vol. 108, pp. 4940-4945, 2004.

\section{Appendix}

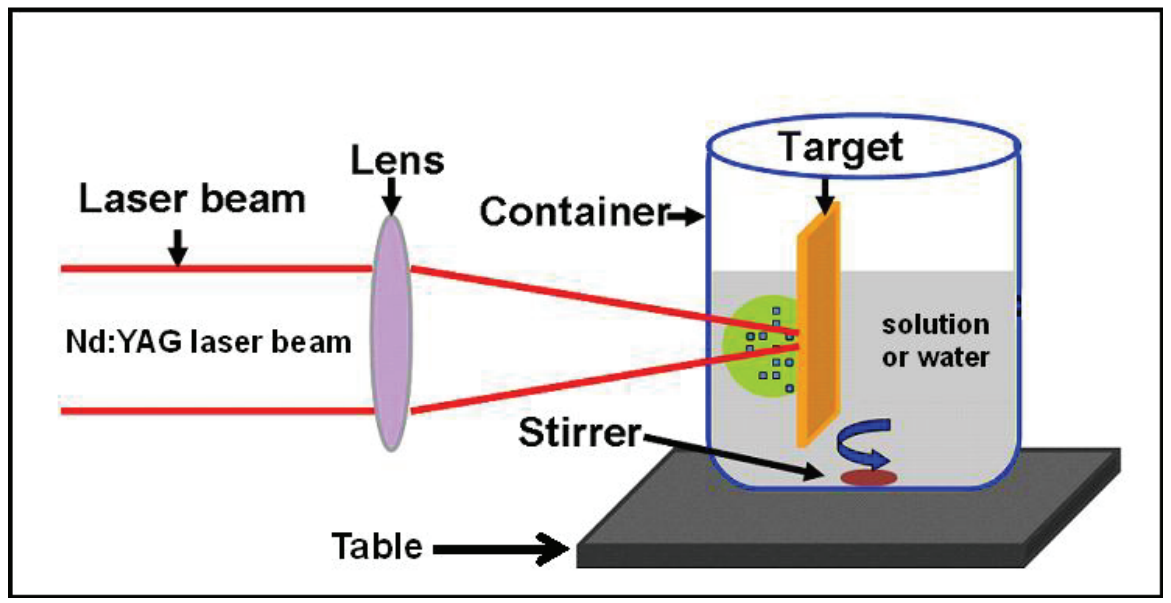

Figure S1. Schematic illustration of pulse laser ablation of a solid target in solution. 


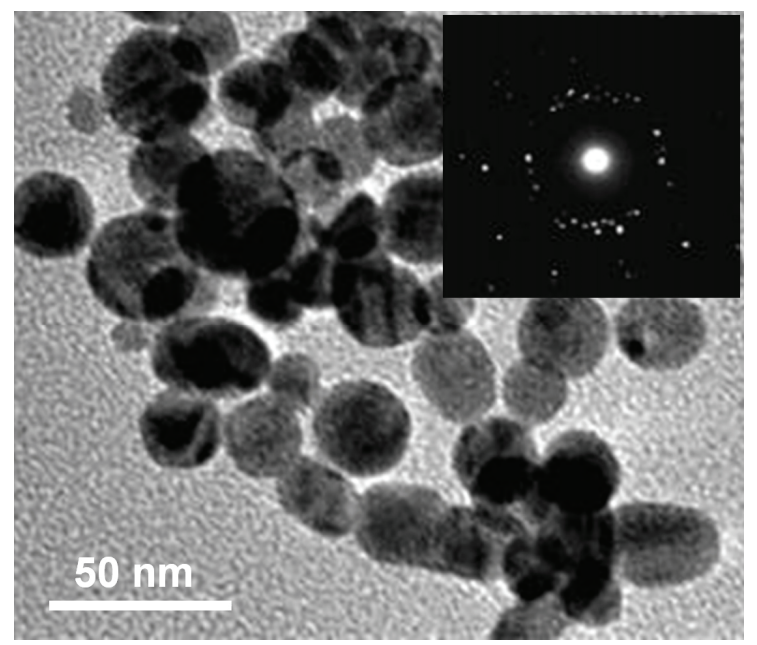

Figure S2. TEM image of products induced by ablation of Au target in the water. The inset is the selected area electronic diffraction of the particles, showing formation of Au NPs with the size below 20nm.

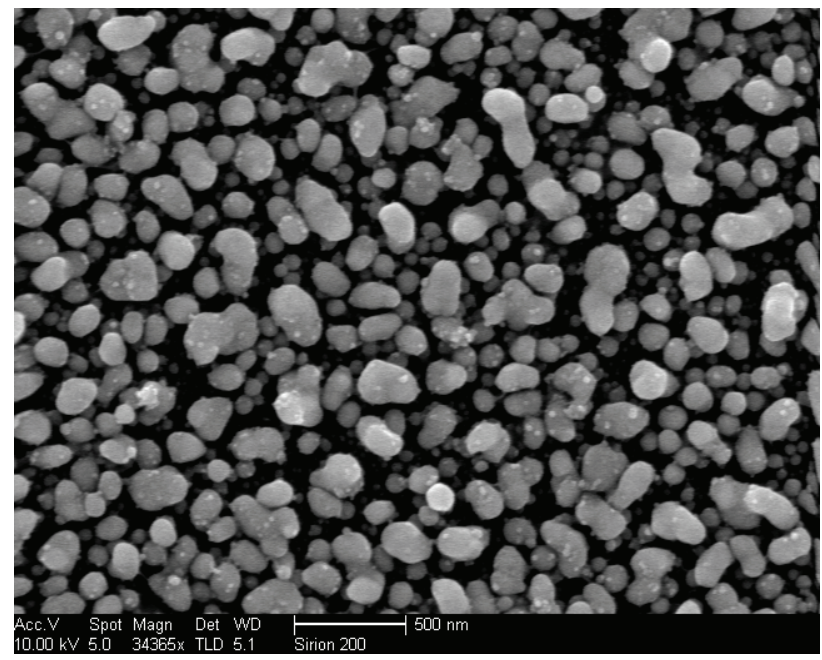

Figure S3. FESEM image for the carbon-wrapped Au NPs' film on ITO glass induced by electrophoretic deposition.

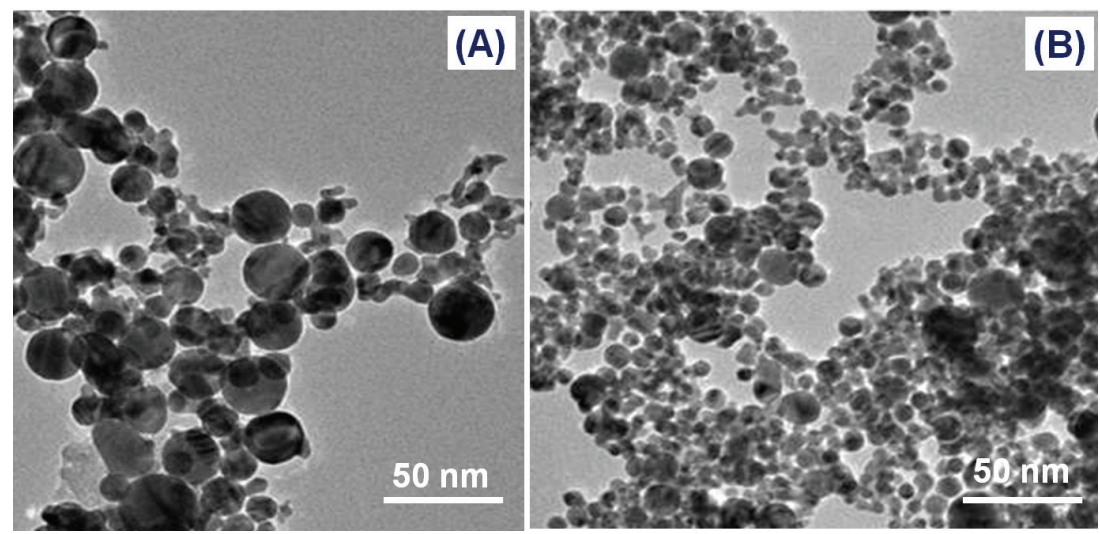

Figure S4. The TEM images of the products obtained after laser ablation of Au target in the toluene-contained ethanol solution using different laser powers. (A): $40 \mathrm{~mJ} /$ plulse. (B):100 mJ/plulse. 